\title{
Exploring Virtual Reality in Construction, Visualization and Building Performance Analysis
}

\author{
M. Al-Adhami ${ }^{\mathrm{a}}$ L. Ma ${ }^{\mathrm{a}}$ and S. Wu $\mathbf{u}^{\mathrm{a}}$ \\ ${ }^{a}$ School of Art, Design and Architecture, University of Huddersfield, UK \\ E-mail: Mus.Adhami@hud.ac.uk, L.Ma@hud.ac.uk, S.Wu@hud.ac.uk
}

\begin{abstract}
In the past two decades, the Architecture, Engineering and Construction (AEC) industry has investigated different approaches to improve communication among project parties, including Virtual reality (VR) however these approaches have not been widely adopted by the industry.

Today, the tremendous advancements in technologies and computer hardware have potentially improved the current approaches and enabled a significant enhancement of user experience of Virtual reality $(V R)$ devices. Based on that the researchers have conducted a review to investigate the global VR applications research in (AEC) community in 20152017 to understand the status and the trend of immersive virtual reality (IVR) research in the world using these affordable devices.

This paper also presents a result of an experiment to integrate three different types of AEC digital modeling data and proposed workflows for IVR applications in construction, visualization and building performance analysis. The experiment deals with construction simulation, rapid generation of the VR scene for existing building and airflow visualization. Several workflows investigated game engine and VR tools have been used.
\end{abstract}

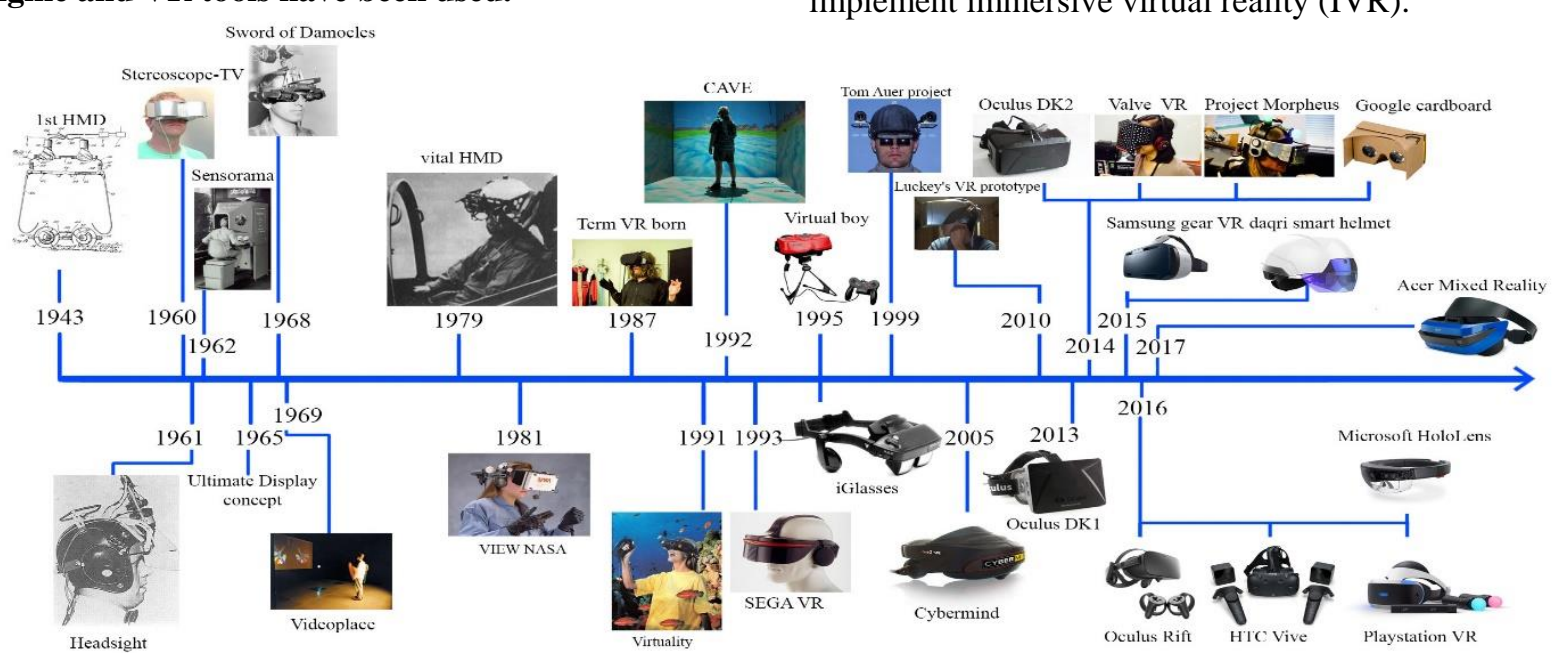

Figure 1. Development of VR headset
Keywords -

Virtual reality; Laser scanning; Building performance simulation; Visualization

\section{Introduction}

The Virtual reality (VR) technology has emerged in various domains in the past two decades. It enables the users to immerse themselves in a computer-generated environment which simulates complex, real situations and contexts[1]. The experience of wearing the VR headset has been significantly enhanced due to the tremendous development of computer hardware, central processing unit (CPU), graphics processing units (GPU), data storage et al. Also, these devices have become more affordable. In fact, any business considers computer graphics and $3 \mathrm{~d}$ modeling will be affected by this technology[2]. The Architecture, Engineering, and Construction (AEC) industry heavily rely on digital modeling, visual communication and simulation[3]. This has attracted the research interests to investigate and applying VR technology to AEC applications.

This paper presented a review of the development of VR technology and their uses in AEC industry through literature review, and conduct an experiment to implement immersive virtual reality (IVR). 


\section{A review of $\mathrm{VR}$ applications in AEC industry}

The research compiled the timeline below that demonstrates the development of VR devices Figure 1. The first head mounted display (HMD) was created by McCollum in 1940s. His idea was to display noninteractive films supporting visual display without tracking motion, but there was no integration of computer and image generation. In the late1980s, the term VR was coined by Jaron Lanier. It combines the devices with video imaging and computer graphics[4]. Now, many headsets have been devised which enable human interaction with computers using motion controllers, depth-sensing camera and natural user interfaces.

This review analyzed all articles related to VR applications since 2015 in the Web of Science core collection database. The review centred on architectural design, construction and building technology. A total of 2,790 paper was found. Figure 2 shows the number of research articles published by authors from different regions. Only the regions that have at least 27 publications are listed here. The researchers from the USA (756), England (286), China (224) and Germany (224) have made the most significant contributions in these areas. Also, researchers for the USA have widely collaborated with researchers from other countries (presented as the thickness of the links between the nodes).

Based on an analysis of the keywords of the 2,790 bibliographic, only 89 articles have been identified that are most related to VR applications in the AEC industry. The keywords used in these articles are shown Figure 3.

The keywords were clustered into five groups (shown

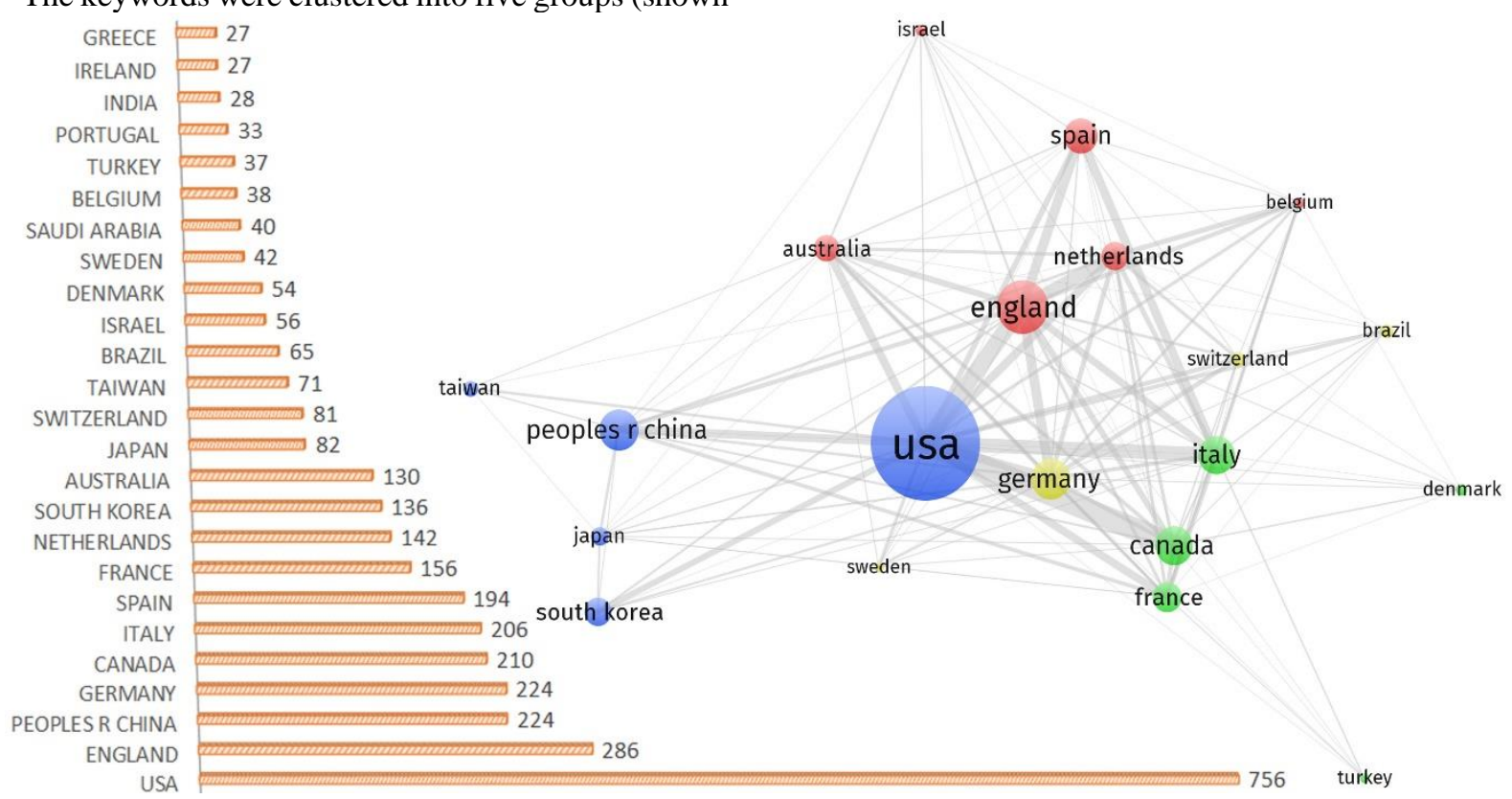

Figure 2 Number of related publications by researchers from different regions in different colours in the figure). They are also listed in Table 1.

Table 1 Clusters of keywords used in publications related to VR applications in AEC industry

\begin{tabular}{cc}
\hline Group & Keywords \\
\hline & Architectural design \\
BIM & Design \\
& Environment \\
& GIS \\
& System \\
& Visual geographic \\
& environment \\
& Visualisation \\
\hline & Augmented reality \\
& Construction \\
& Dwelling \\
& Education \\
& Mixed reality \\
& Safety \\
& Technology \\
& Unity \\
& Architecture \\
& Cultural heritage \\
& Perception \\
& Space perception \\
& Virtual reality \\
\hline \multirow{3}{*}{3} & Evacuation \\
& Games \\
\hline \multirow{2}{*}{5} & Construction safety \\
& Management \\
\hline &
\end{tabular}




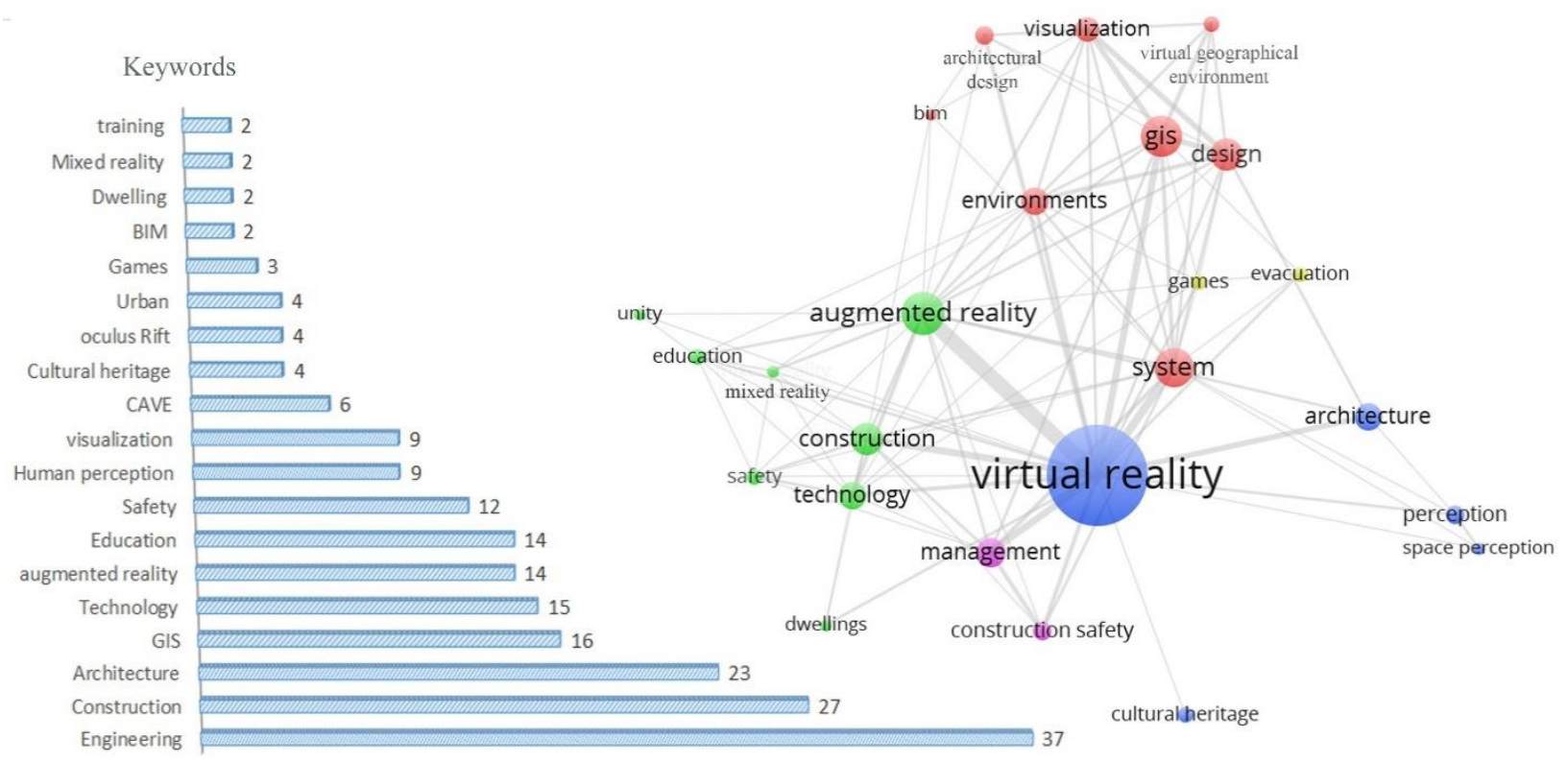

Figure 3. Keywords used in applications of VR in AEC industry

The group 1 shows that BIM-enabled VR has been widely used in architectural design and urban planning (GIS system) for visualization purpose; the group 2 shows that VR together with augmented reality and mixed reality technologies have been widely used for construction safety education; group 3 shows that VR has been used for investigation of space perception in cultural heritage; group 4 shows that VR has been used for education through games; group 5 shows that VR has also been implemented in construction safety management.

Most of the researchers mainly utilised the nonimmersive VR devices in their work. These devices mainly provide visualisation capability, while the immersive VR devices enable more advanced human interaction with the computer. Nine research papers [3, 5-12] using immersive VR are shown in Table 2.

\section{Experiment for establishing a workflow of IVR applications}

The experiment based on three types of data:

1. 4D construction simulation in VR

2. Rapid generation of the VR scene

3. Airflow visualization in VR

Table 2 Research using Immersive VR

\begin{tabular}{cc}
\hline Group & Keywords \\
\hline 2017 & Diegetic user interfaces for virtual environments with HMDs: a user experience study with oculus rift \\
2017 & vConnect: perceive and interact with real world from CAVE \\
2016 & Design and Evaluation of Data Annotation Workflows for CAVE-like Virtual Environments \\
2016 & A virtual reality integrated design approach to improving occupancy information integrity for closing \\
& the building energy performance gap \\
2016 & Construction and Evaluation of an Ultra-Low Latency Frameless Renderer for VR \\
2015 & Neurophysiological correlates of embodiment and motivational factors during the perception of \\
& virtual architectural environments \\
2015 & The effect of dangerous goods transporters on hazard perception and evacuation behavior - A virtual \\
& reality experiment on tunnel emergencies \\
2015 & Mobile, Low-Cost, and Large-Scale Immersive Data Visualization Environment for Civil \\
2015 & Engineering Applications \\
& Immersive virtual environments versus physical built environments: A benchmarking study for \\
building design and user-built environment explorations
\end{tabular}




\subsection{Construction simulation}

We prepared the BIM model of the Queen Street Studio (QSS) at the University of Huddersfield using Revit; convert the model data from RVT to FBX file format; then import the FBX file in Unity (a game engine). It was found that the texture of the BIM model was missing in Unity, so the modeller needed to add the texture layers in Unity again.
For the 4D simulation, we used Autodesk navisworks manager to divide the BIM model into parts that represent the construction batches. Then, we defined the construction schedules using timelines, as is shown in Figure 4. The TimeLiner data was exported from naviswork to $3 \mathrm{ds}$ max and then the animation data with geometries was exported to Unity. Finally, the immersive VR application was generated from Unity, as is shown in Figure 5.

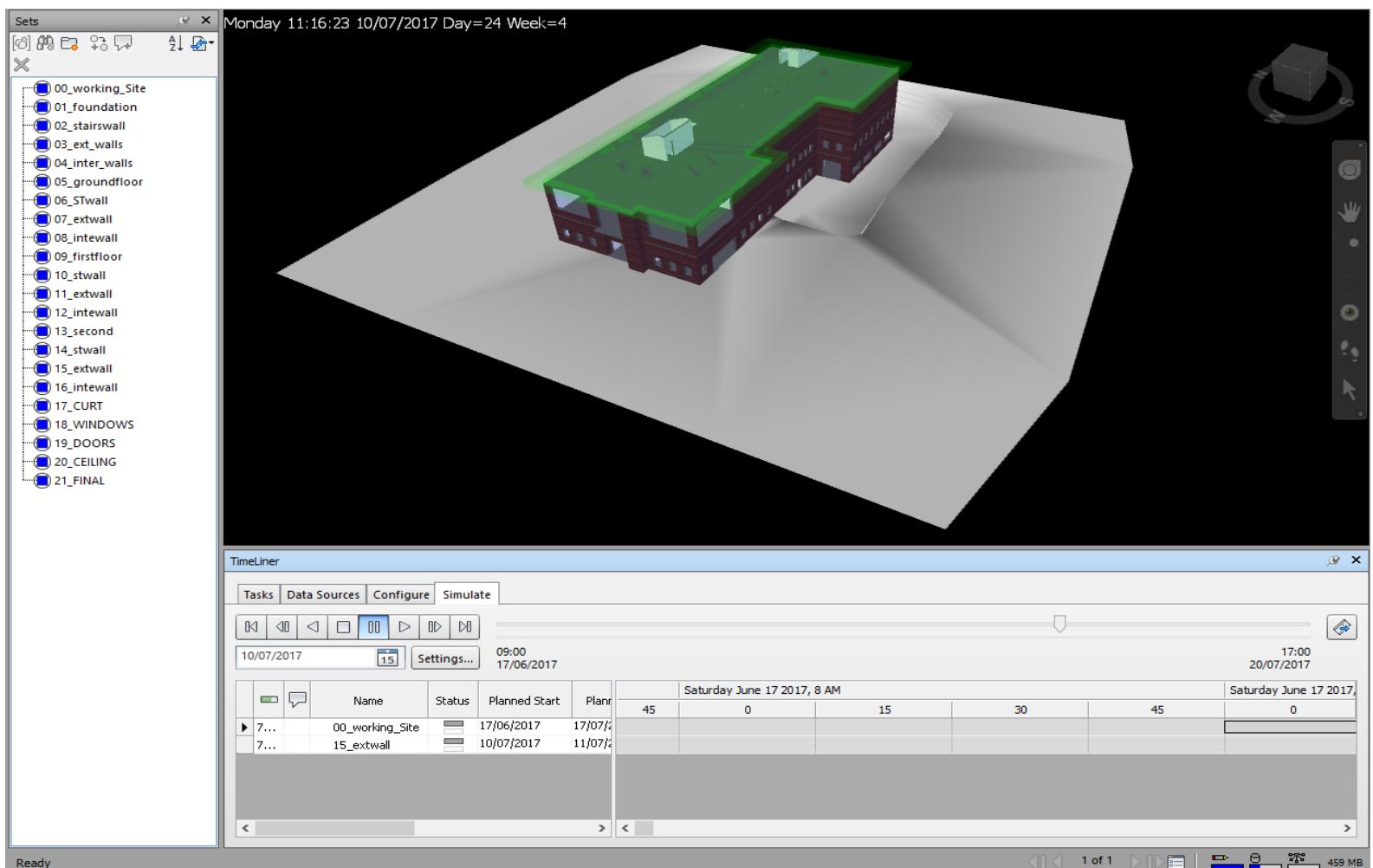

Figure 4. The BIM model and construction sequence of the Queen Street Studio

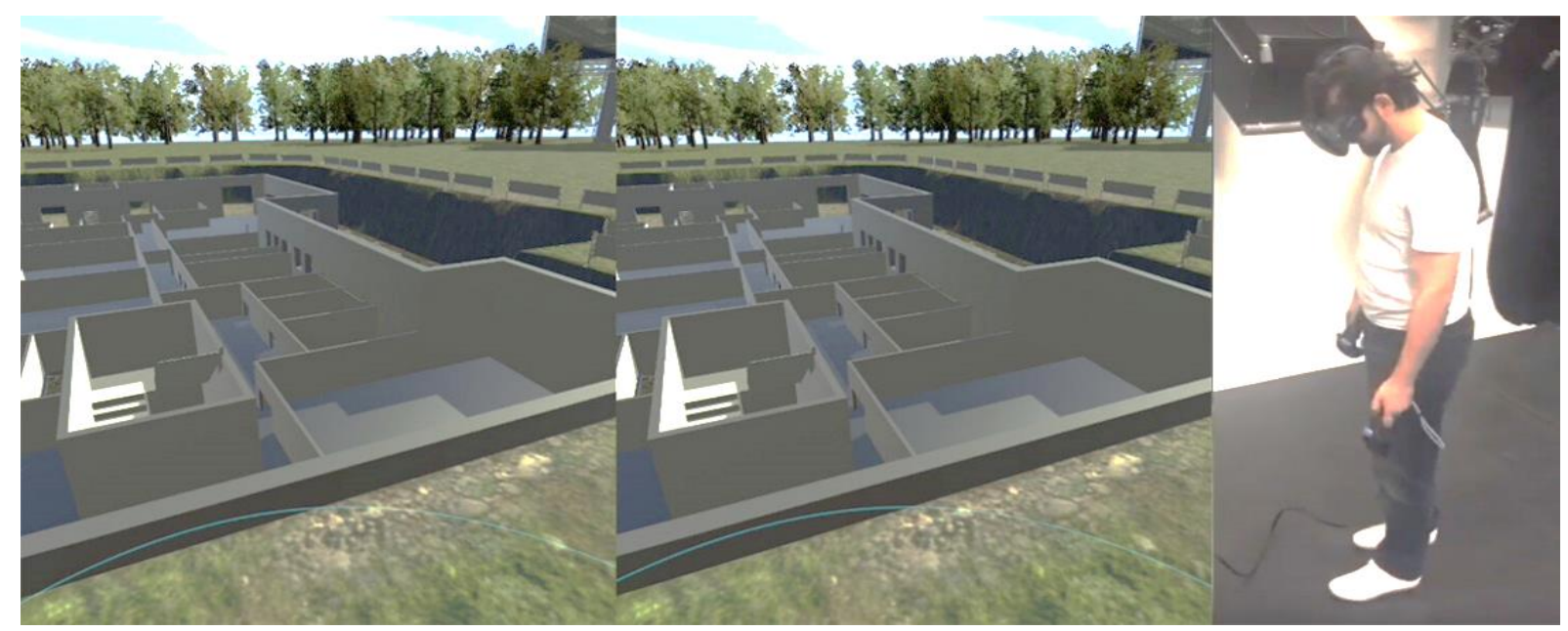

Figure 5. Construction simulation in immersive VR 


\subsection{Rapid generation of VR scenes}

The Terrestrial Laser Scanner is a state-of-the-art survey technology. We used the FARO Focus scanner to capture the 'as-is' state of four rooms in the QSS building, as is shown in Figure 6. The rooms were chosen according to the space area, type of lighting, and the function (shown in Table 3).
Then, we generated the mesh model of the room using the collected point cloud data and then import it to Unity and built the VR application, as is shown in Figure 7. We also import the point cloud directly to Unity and built the VR application as is shown in Figure 8. The mesh model has a better visualization while it requires more process time.
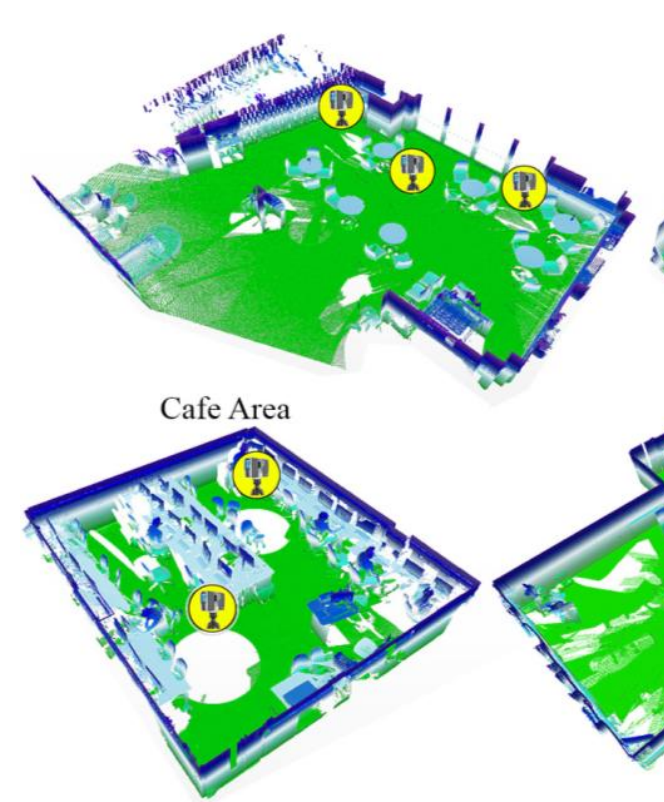

PC Lab

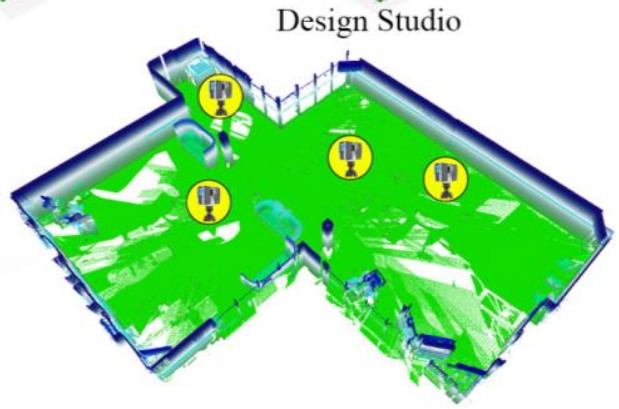

Design Centre

Figure 6. The scanned four rooms in the Queen Street Studio

Table 3. The condition of the rooms chosen for experiments

\begin{tabular}{|c|c|c|c|c|c|}
\hline Space Name & Floor & Type & Area(m2) & $\begin{array}{c}\text { Lighting / windows } \\
\text { Orientation }\end{array}$ & $\begin{array}{c}\text { Scan } \\
\text { stations }\end{array}$ \\
\hline Café Area & Ground Floor & Public & 62 & Daylighting - SE & Three \\
\hline Design Centre & Ground Floor & Semi public & 188 & Artificial - S & Four \\
\hline PC Lab & Basement & Private & 58 & Artificial - none & Two \\
\hline Design Studio & First Floor & Private & 88 & Mixed - SW & Three \\
\hline
\end{tabular}

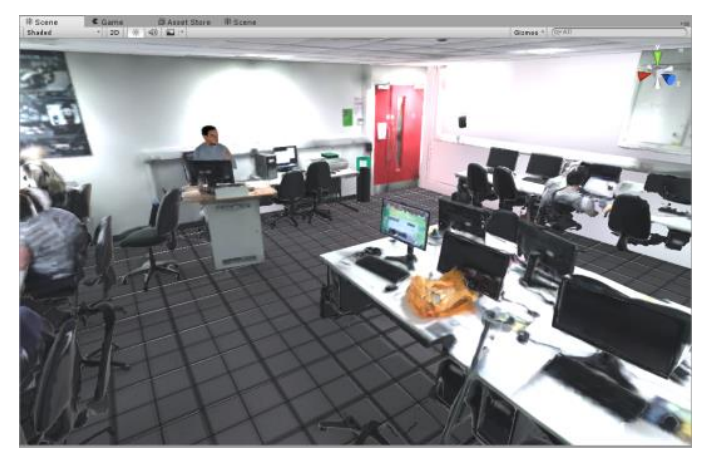

Figure 7. Mesh object in Unity

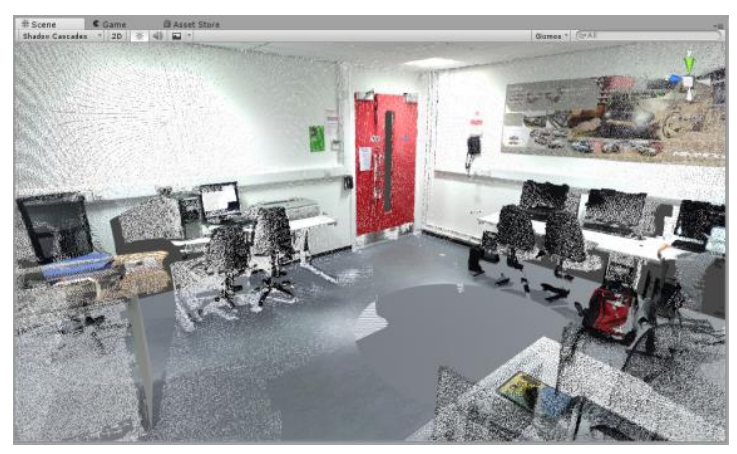

Figure 8. Point cloud data in Unity 


\subsection{Rapid generation of VR scenes}

We used Autodesk CFD to perform CFD simulation of the PC lab at the QSS building. Simulation parameters include: Air Changes per hour is (15) and Supply temperature is $\left(20^{\circ} \mathrm{C}\right)$. The results were exported as CSV files which contain the information of velocity, temperature, pressure and density in the simulated space, as is shown in Figure 9.
CFD data was then imported to 3ds Max to generate geometries for visualizing the velocity, temperature, and pressure. There are several representations of CFD data such as splines, planes, arrows, etc.

We set airflow data as splines with temperature colour. The data was then exported as FBX file and imported as a new asset in Unity. Finally, the VR application was built and visualised in the headset, as is shown in Figure 10.
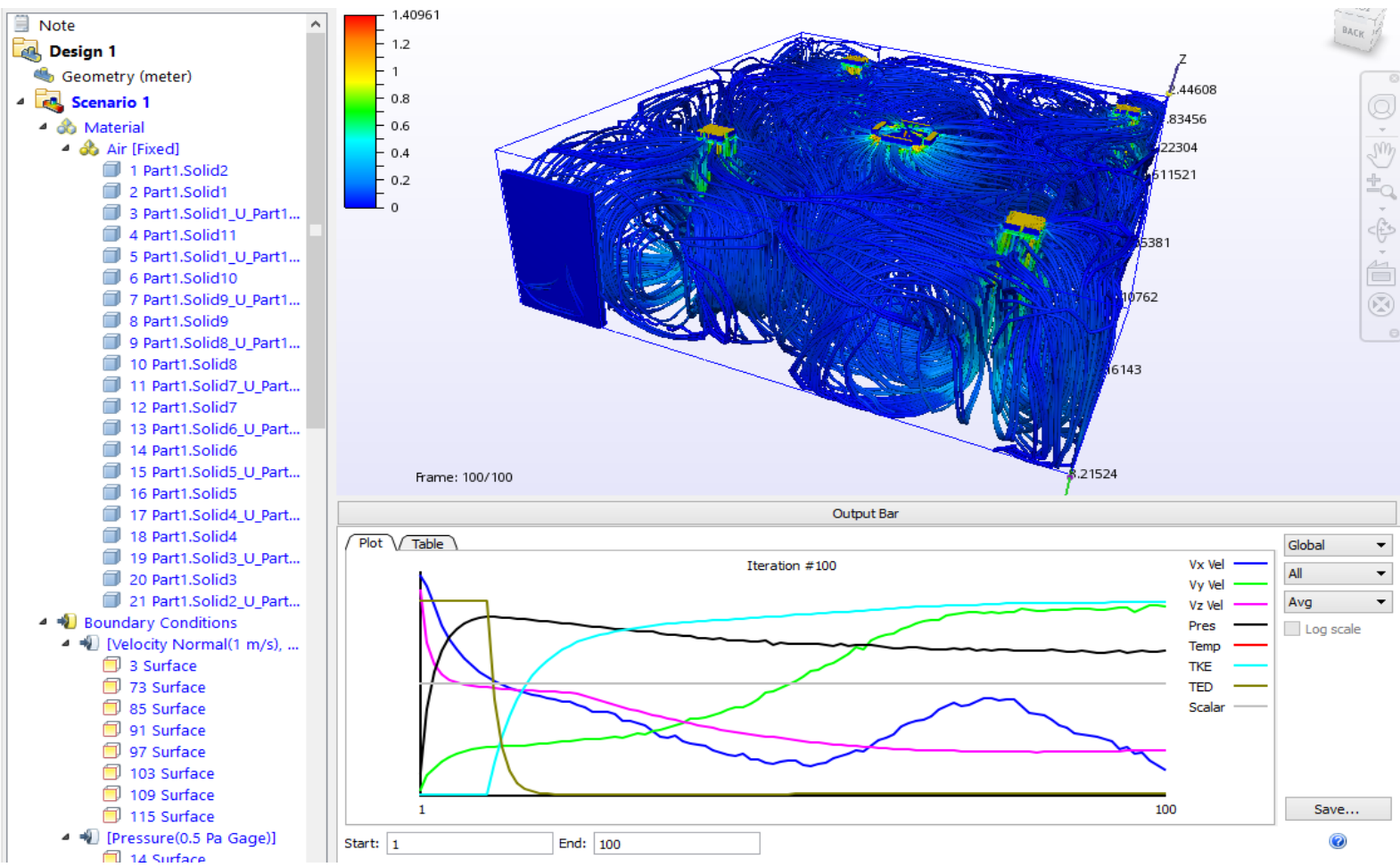

Figure 9. Computational Fluid Dynamics (CFD) simulation of the PC Lab at QSS

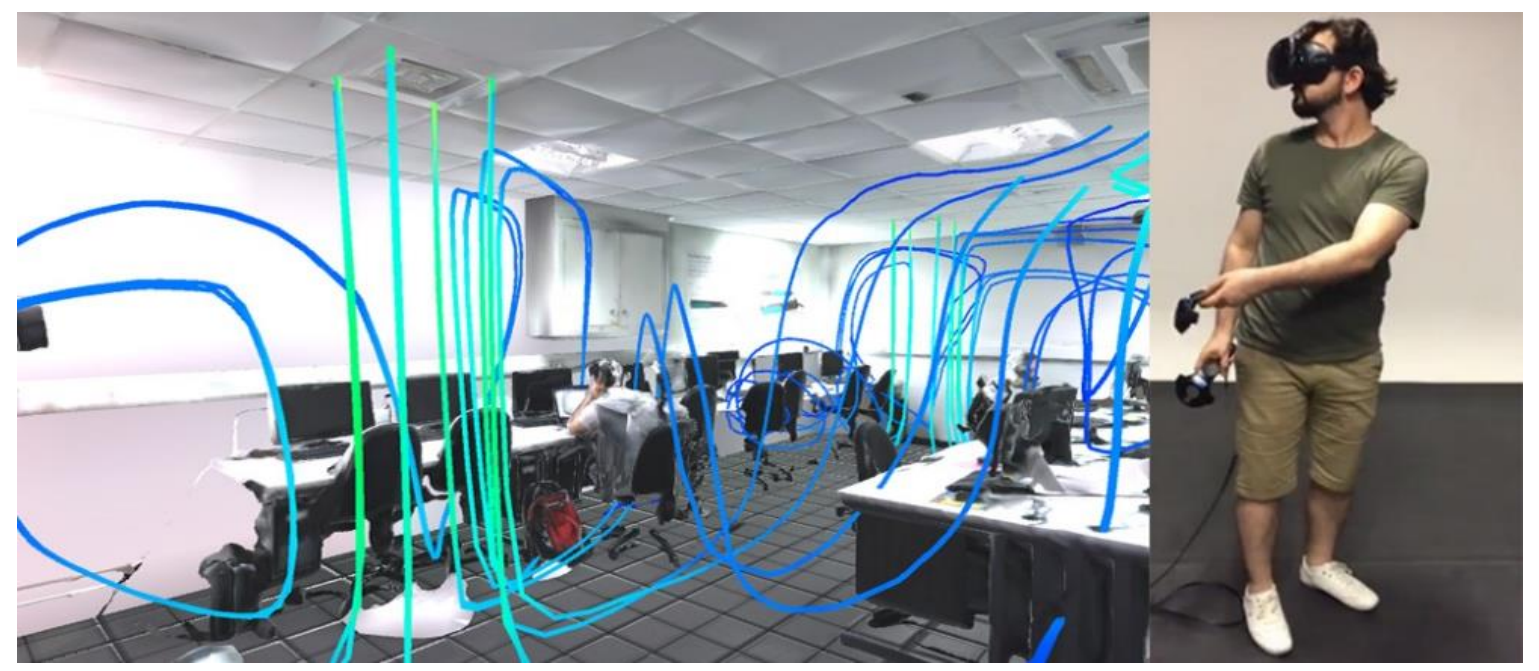

Figure 10. Visualisation of the airflow in immersive VR 


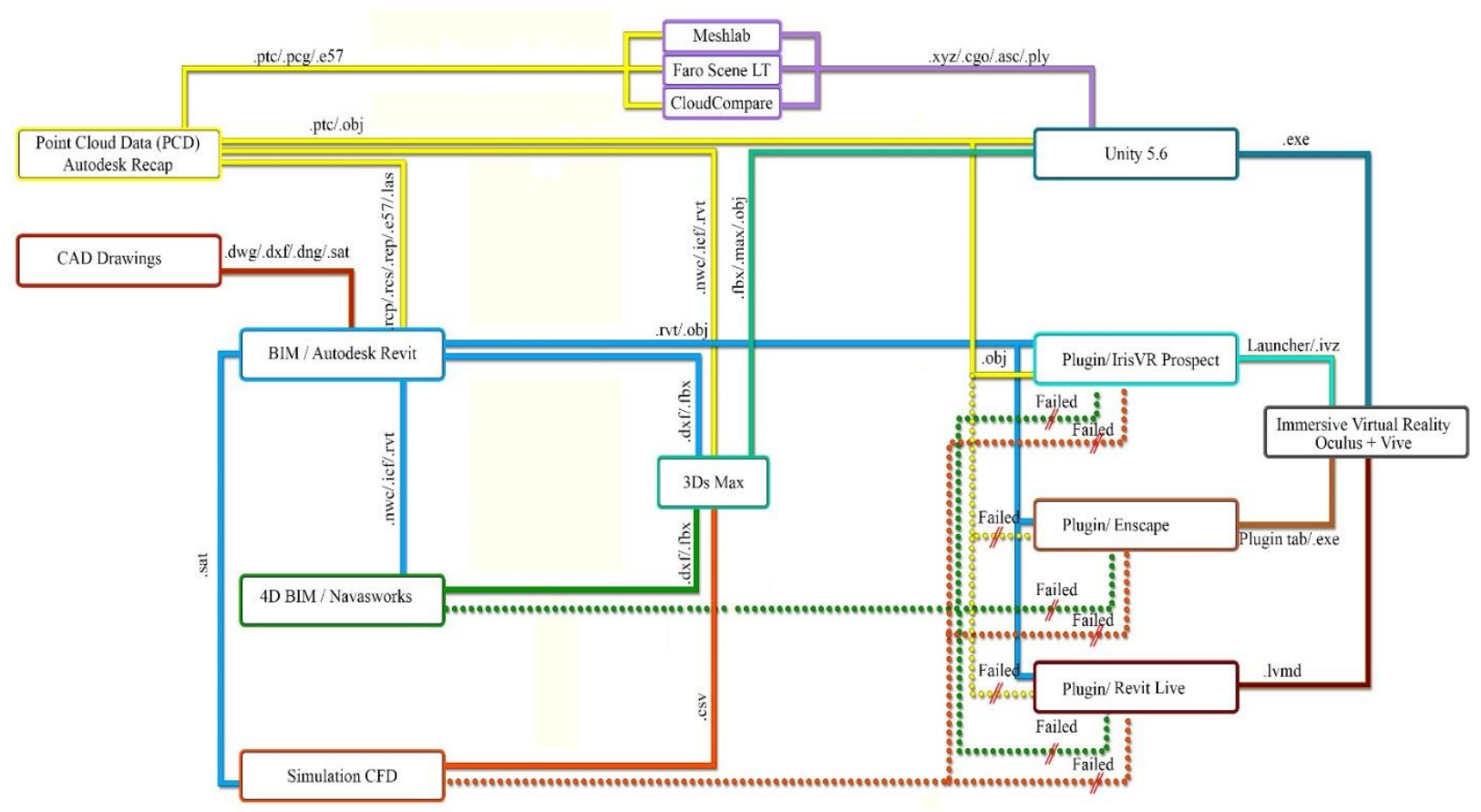

Figure 11. The interoperability of VR applications

\section{Summary}

This paper reviewed the development of VR devices and literature of VR applications for AEC industry. It also demonstrated three workflows for construction simulation, VR scene generation, and building performance analysis. The literature has shown VR technology was successfully applied in many fields, however, the use of immersive virtual reality (IVR) still not adopted widely, AEC industry is facing many challenges starting from concept design, construction and operation, today VR technology can be a valuable tool to solve some of these challenges.

There are many tools can be used to create VR applications and it is important to understand their usages, functions and interoperability. Figure 11 shows how to exchange data between different tools. This can help others to select the right tools for using VR in their business processes. However, there are few things we need to consider to develop a sophisticated VR experience, 1) knowing the user, the problem, outline the expectation, and the final goal, 2) VR platform (desktop or mobile) and identify the content of creation in reference to VR experience. 3) Integration method and developing time. In addition, the model needs to be created with fewer polygons so that the model will not be too heavy to be loaded into the VR platform and also get rid of unwanted geometries to avoid overload objects. It is highly recommended to give a unique name to each object in the model and group some of them; this helps organize the work through getting the information faster especially in big projects, and make things less complicated if there is a need to change, edit or replace some object.

\section{References}

[1] Diemer J.Alpers G W.Peperkorn H M.Shiban Y and Mühlberger A. The impact of perception and presence on emotional reactions: a review of research in virtual reality. Frontiers in psychology, 6: 26, 2015.

[2] Linowes J. Unity Virtual Reality Projects, (Issue). Packt Publishing Ltd, 2015.

[3] Heydarian A.Carneiro J P.Gerber D.Becerik-Gerber B.Hayes $\mathrm{T}$ and Wood W. Immersive virtual environments versus physical built environments: A benchmarking study for building design and userbuilt environment explorations. Automation in Construction, 54: 116-126, 2015.

[4] Steinicke F. Being Really Virtual, (Issue). Springer, 2016.

[5] Salomoni P.Prandi C.Roccetti M.Casanova L.Marchetti L and Marfia G. Diegetic user interfaces for virtual environments with HMDs: a user experience study with oculus rift. Journal on Multimodal User Interfaces, 11(2): 173-184, 2017.

[6] He Y F.Zhang Z Y.Nan X M.Zhang N.Guo F.Rosales E and Guan L. vConnect: perceive and interact with real world from CAVE. Multimedia 
Tools and Applications, 76(1): 1479-1508, 2017.

[7] Pick S.Weyers B.Hentschel B and Kuhlen T W. Design and Evaluation of Data Annotation Workflows for CAVE-like Virtual Environments. Ieee Transactions on Visualization and Computer Graphics, 22(4): 1452-1461, 2016.

[8] Niu S Y.Pan W and Zhao Y S. A virtual reality integrated design approach to improving occupancy information integrity for closing the building energy performance gap. Sustainable Cities and Society, 27: 275-286, 2016.

[9] Friston S.Steed A.Tilbury S and Gaydadjiev G. Construction and Evaluation of an Ultra Low Latency Frameless Renderer for VR. Ieee Transactions on Visualization and Computer Graphics, 22(4): 1377-1386, 2016.

[10] Vecchiato G.Jelic A.Tieri G.Maglione A G.De Matteis F and Babiloni F. Neurophysiological correlates of embodiment and motivational factors during the perception of virtual architectural environments. Cognitive Processing, 16: S425S429, 2015.

[11] Kinateder M.Gromer D.Gast P.Buld S.Muller M.Jost M.Nehfischer M.Muhlberger A and Pauli P. The effect of dangerous goods transporters on hazard perception and evacuation behavior - A virtual reality experiment on tunnel emergencies. Fire Safety Journal, 78: 24-30, 2015.

[12] Hayden S.Ames D P.Turner D.Keene T and Andrus D. Mobile, Low-Cost, and Large-Scale Immersive Data Visualization Environment for Civil Engineering Applications. Journal of Computing in Civil Engineering, 29(6): 10, 2015. 